\title{
The 3-D Commission: Forging a Transdisciplinary Synthesis at the Intersection of Social Determinants of Health, Data, and Decision-making
}

\author{
Salma M Abdalla • Sandro Galea
}

Accepted: 7 May 2021 / Published online: 19 August 2021

(C) The New York Academy of Medicine 2021

There is a growing recognition of the role social determinants of health $(\mathrm{SDoH})$ - the conditions in which people live, grow, learn, work, play, and age - play in shaping the health of individuals and populations [1]. Concurrently, the past two decades have witnessed an expansion of digital technologies that allow for the collection of data on our biology and on the forces in the world around us that affect our lives with far greater granularity than was previously possible. This expansion is often referred to as "big data" and has paved the way for new opportunities to document, measure, and analyze the forces that shape individual and population health.

The science of the SDoH and the growth of "big data" have largely developed separately, with relatively little interaction. It is not difficult, however, to envision how they can inform each other and, ultimately, guide decision-making to the end of improving health outcomes.

The Rockefeller Foundation, Boston University Commission on Determinants of health, Data, and Decision-making (3-D Commission) aims to accelerate

S. M. Abdalla $(\bowtie) \cdot$ S. Galea

Rockefeller Foundation-Boston University 3-D Commission on Determinants, Data, and Decision-making, Boston, USA

e-mail: abdallas@bu.edu

S. Galea

e-mail: sgalea@bu.edu

S. M. Abdalla $\cdot$ S. Galea

Department of Epidemiology, Boston University School of Public Health, Boston, MA, United States this transdisciplinary synthesis through bringing together a diverse group of 25 experts from around the globe [2]. The formation of the 3-D Commission was grounded in the premise that SDoH matter, that data on SDoH can help inform our understanding of how they matter, and that combining these two intellectual strands can help inform decision-making to the end of improving the health of individuals and populations [3]. The 3-D Commission convened for three meetings that were complemented by nine smaller working group meetings. The final 3-D Commission report - which will be launched during the United Nations General Assembly in September 2021 - will reflect the Commission deliberations, put forward a set of principles to advance both the intellectual and practical agenda of the Commission, and propose actionable recommendations for decision-makers.

\section{Contributions in This Special Issue}

A core goal of the 3-D Commission is to engage with, and advance, scholarship at the intersection of SDoH, data, and decision-making. To that end, this supplement adds to the literature eight studies that, while using methods to assess different health indicators, all aim to answer questions around how we may use data on SDoH to inform decision-making. Taken together, these studies offer important insights on the status of the scholarship that aim to connect these different fields.

Biermann et al. summarize the evolution of the social framing of health over the past few decades and describe 
how SDoH are conceptualized and contextualized differently at the global, national, and local levels [4]. The paper argues for analyzing and understanding $\mathrm{SDoH}$ relative to the contexts in which they are experienced and problematizes the gap in data on different $\mathrm{SDoH}$ across contexts. Mwoka et al. demonstrate the importance of context around $\mathrm{SDoH}$ through documenting the extent to which housing policies address health and wellbeing in three countries at different levels of the wealth spectrum: Singapore, the UK, and Kenya [5]. The paper suggests that demographic population composition, political ideologies, legal frameworks, and presence (or absence) of adequate, quality, timely, reliable, robust data systems, all have strong implications of the type of housing policies developed and implemented in each country.

Two papers examine the landscape of data sources on SDoH in middle-income countries. Torres et al. conduct an overarching review of the sources and uses of data on SDoH in Kenya and the Philippines while Thapa et al. examine the data sources for assessing depression and its SDoH in Brazil and India [6, 7]. Both papers argue that despite the emergence of new data sources (e.g., social media or geographic information system), there is limited evidence of the use of such data to either study $\mathrm{SDoH}$ in general or study the determinants of depression. Both papers find that even in the rare occasions new data sources are used, they often supplement the use of traditional sources (e.g., surveys) of data. The papers suggest that the limited use of new sources could be due to several factors including the absence of standardized indicators of $\mathrm{SDoH}$, inadequate trust in data collection methods, and limited infrastructure to pool, analyze, and develop new data sources.

Martins et al. suggest two opportunities to explicitly integrate data on $\mathrm{SDoH}$ in commonly cited conceptual approaches for policy-making processes [8]. First, data on SDoH can provide appropriate visibility and credibility to health issues, making them part of the consideration on policy-making processes. Second, the use of the SDoH framing can more effectively attract the attention of a diverse group of decision-makers with the power to allocate resources, which can help improve the health of populations. The conclusions of this paper are echoed in the findings of Babajide et al. which examine the extent to which data on $\mathrm{SDoH}$, equity, and multi-sectoral approaches are incorporated into local politics and decision-making processes around Bus Rapid Transit (BRTs) systems in three megacities,
Lagos, Bogotá, and Beijing [9]. This paper finds that all megacities do not afford adequate attention to health in their agenda-setting for BRTs-related policies, despite having transportation policies that aim to support health in principle.

The supplement also includes two scoping reviews on the available literature linking big data on food and energy to decision-making. The majority of studies identified by Biermann et al. assess the link between food and food systems and big data, while hypothesizing about how insights from these analyses could potentially inform decision-making [10]. Only two studies in the scoping review use big data on food and food systems to inform decision-making directly. A number of themes emerge in the review by Maani et al. on data on energy and decision-making including the link between energy consumption and economic development, the role of inequality in understanding and predicting harms and benefits associated with energy production and use, and the lack of available data on LMICs, particularly local contexts in LMICs [11]. Both scoping reviews highlight the importance of context, both between and within countries, in shaping the type and availability of data on SDoH that can be used as meaningful evidence to inform decision-making.

\section{Themes That Advance the 3-D Commission Agenda}

Across the articles in this supplement, two central themes emerge. First, context matters and is critical to understanding the particular SDoH relevant to a specific population. As such, there is a need to collect, and use, current and granular data that characterize the full range of SDoH at the national, and local, levels. Developing such an efficient system will require engaging with all available data sources and go beyond traditional surveys. Further, contextualizing data on the national and local levels points towards the importance of adopting a participatory approach to both data collection and decision-making that centers the communities from which the data are being collected.

Second, there is paucity in the literature and practice of linking SDoH, data, and decision-making processes. This highlights the need for investment in both scholarship and practice approaches that facilitate the incorporation of data on $\mathrm{SDoH}$ in decision-making to the end of improving the health of individuals and populations. This also points to the need to advance scholarship and 
practice that ensure that health is a consideration in investment decisions in all sectors. Ultimately, healthy societies are the outcome of a cross-sectoral approach to health, and that goal is not achievable without a broader based approach to health, extending beyond the traditional remit of healthcare.

\section{Looking Ahead}

This supplement offers a window into the state of the scholarship at the intersection of SDoH, data, and decision-making. In so doing, it also demonstrates the need for more research in the area that can lead to a transdisciplinary synthesis. Taken together with the 3-D Commission report, this work argues for a more robust engagement with data on the SDoH paving the way for decision-making across sectors that accounts for these determinants. This work aims both to encourage future scholarship and also to nudge us collectively to better data collection about social determinants, and better decision-making for health.

Acknowledgements The Rockefeller Foundation-Boston University 3-D Commission (Grant number: 2019 HTH 024).

\section{References}

1. WHO Commission on social determinants of health. Closing the gap in a generation: health equity through action on the social determinants of health. Geneva; 2008. https://www. who.int/social_determinants/final_report/csdh_ finalreport_2008.pdf. Accessed January 8, 2020.

2. 3-D Commission. https://3dcommission.health/. Accessed May 17, 2021.

3. Galea S, Abdalla SM, Sturchio JL. Social determinants of health, data science, and decision-making: forging a transdisciplinary synthesis. PLOS Med. 2020;17(6):e1003174. https://doi.org/10.1371/journal.pmed.1003174.

4. Biermann O, Mwoka M, Ettman CK, et al. Data, social determinants, and better decision-making for health. $J$ Urban Health. 2021; (this issue)

5. Mwoka M, Biermann O, Ettman CK, et al. Housing as a social determinant of health: evidence from Singapore, United Kingdom and Kenya. J Urban Health. 2021; (this issue)

6. Torres I, Thapa B, Robbins G, et al. Data sources for understanding the social determinants of health: examples from two lower middle-income countries. J Urban Health. 2021; (this issue)

7. Thapa B, Torres I, Koya SF, et al. Use of data to understand the social determinants of depression in two middle-income countries. J Urban Health. 2021; (this issue)

8. Martins DC, Babajide O, Maani N, et al. Integrating social determinants in decision-making processes for health: insights from conceptual frameworks. J Urban Health. 2021; (this issue)

9. Babajide O, Martins DC, Maani N, et al. Improving decision-making for population health in non-health sectors in urban environments: the example of the transportation sector in three megacities. J Urban Health. 2021; (this issue)

10. Biermann O, Koya SF, Corkish C, et al. Food, big data and decision-making: a scoping review. J Urban Health. 2021; (this issue)

11. Maani N, Robbins G, Koya SF, et al. Energy, data, and decision-making: a scoping review. J Urban Health. 2021; (this issue)

Publisher's Note Springer Nature remains neutral with regard to jurisdictional claims in published maps and institutional affiliations. 